Supplement of Biogeosciences, 16, 4535-4553, 2019

https://doi.org/10.5194/bg-16-4535-2019-supplement

(C) Author(s) 2019. This work is distributed under

the Creative Commons Attribution 4.0 License.

(c) (1)

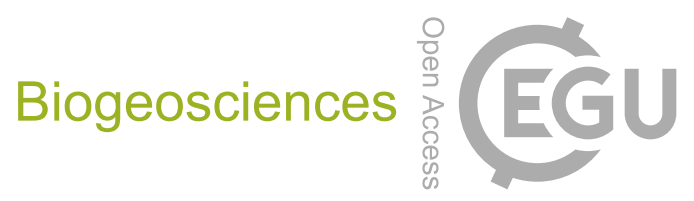

Supplement of

\title{
Comparisons of dissolved organic matter and its optical characteristics in small low and high Arctic catchments
}

Caroline Coch et al.

Correspondence to: Caroline Coch (coch.caroline@gmail.com)

The copyright of individual parts of the supplement might differ from the CC BY 4.0 License. 


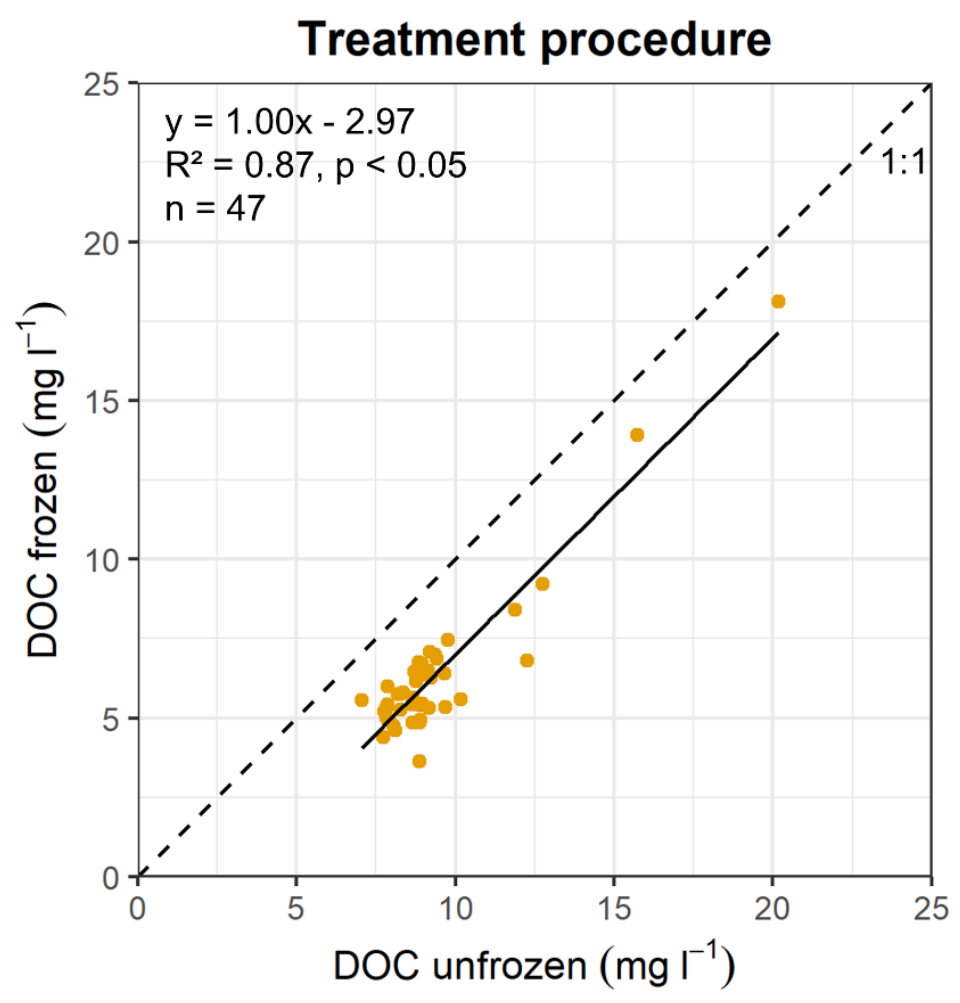

Figure S1: Relationship between different sample treatment procedure in the field for sample duplicates. Those sample duplicates followed two different procedures: (1) samples remaining unfrozen in the field and acidifying after filtration through $0.7 \mu \mathrm{m}$ GF/F 5 filters and (2) samples frozen in the field after filtration through $0.7 \mu \mathrm{m} \mathrm{GF/F} \mathrm{filters,} \mathrm{thawed} \mathrm{and} \mathrm{acidified} \mathrm{upon} \mathrm{return} \mathrm{to} \mathrm{the} \mathrm{lab} \mathrm{in}$ Germany. There is a significant $(p<0.05)$ relationship between both sample procedures, which is why this relationship was used to correct samples that had to be frozen in the field (modified after Coch et al. (2018)) 


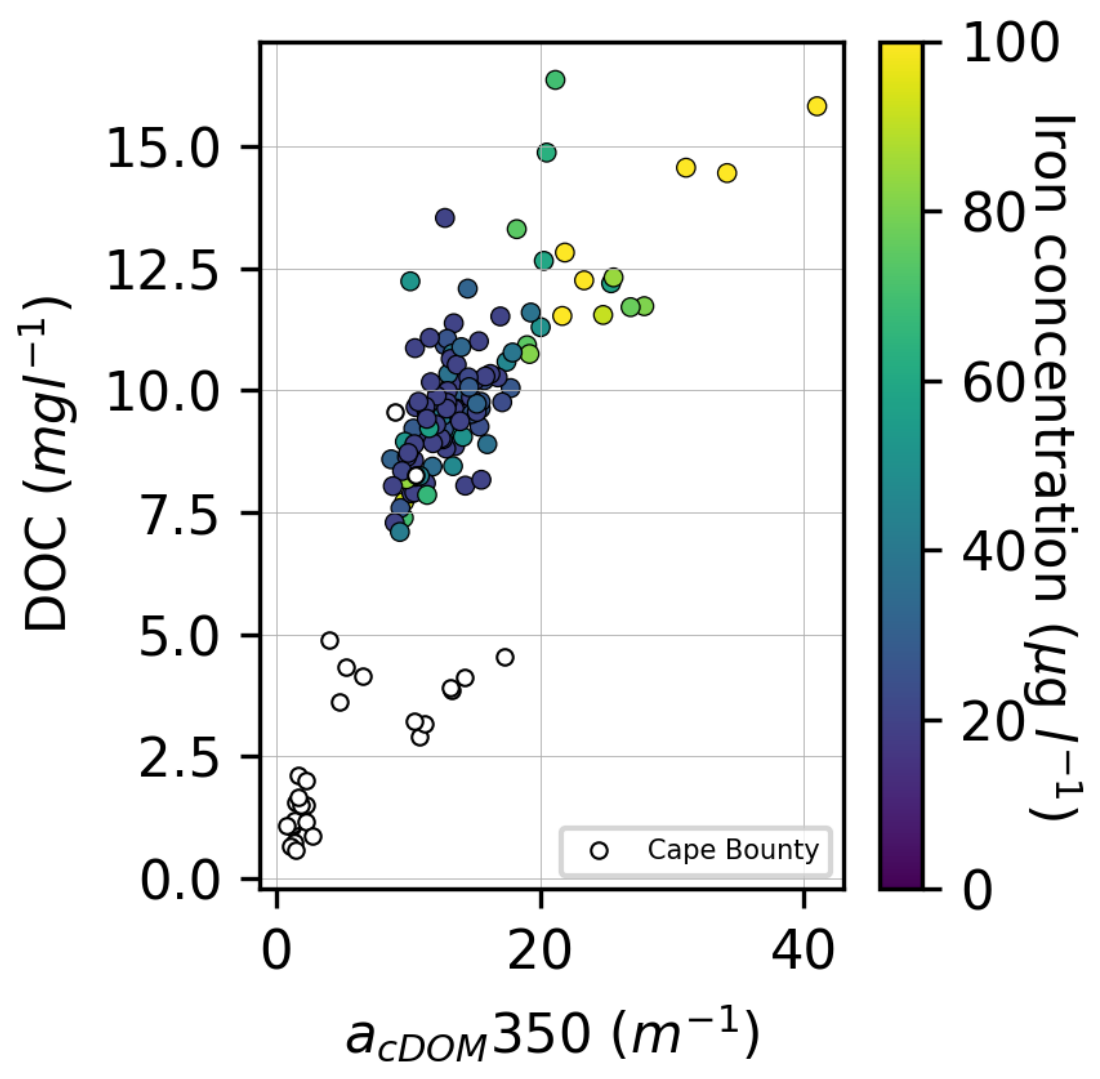

Figure S2. Relationship between acDom350 $\left(\mathrm{m}^{-1}\right)$ and DOC concentration $\left(\mathrm{mg} \mathrm{l}^{-1}\right)$ for our study sites. The colour indicates the measured total iron concentration between 0 and $100 \mu$ l-1 for the Herschel Island samples. No information on iron concentration is available for samples from Cape Bounty. 


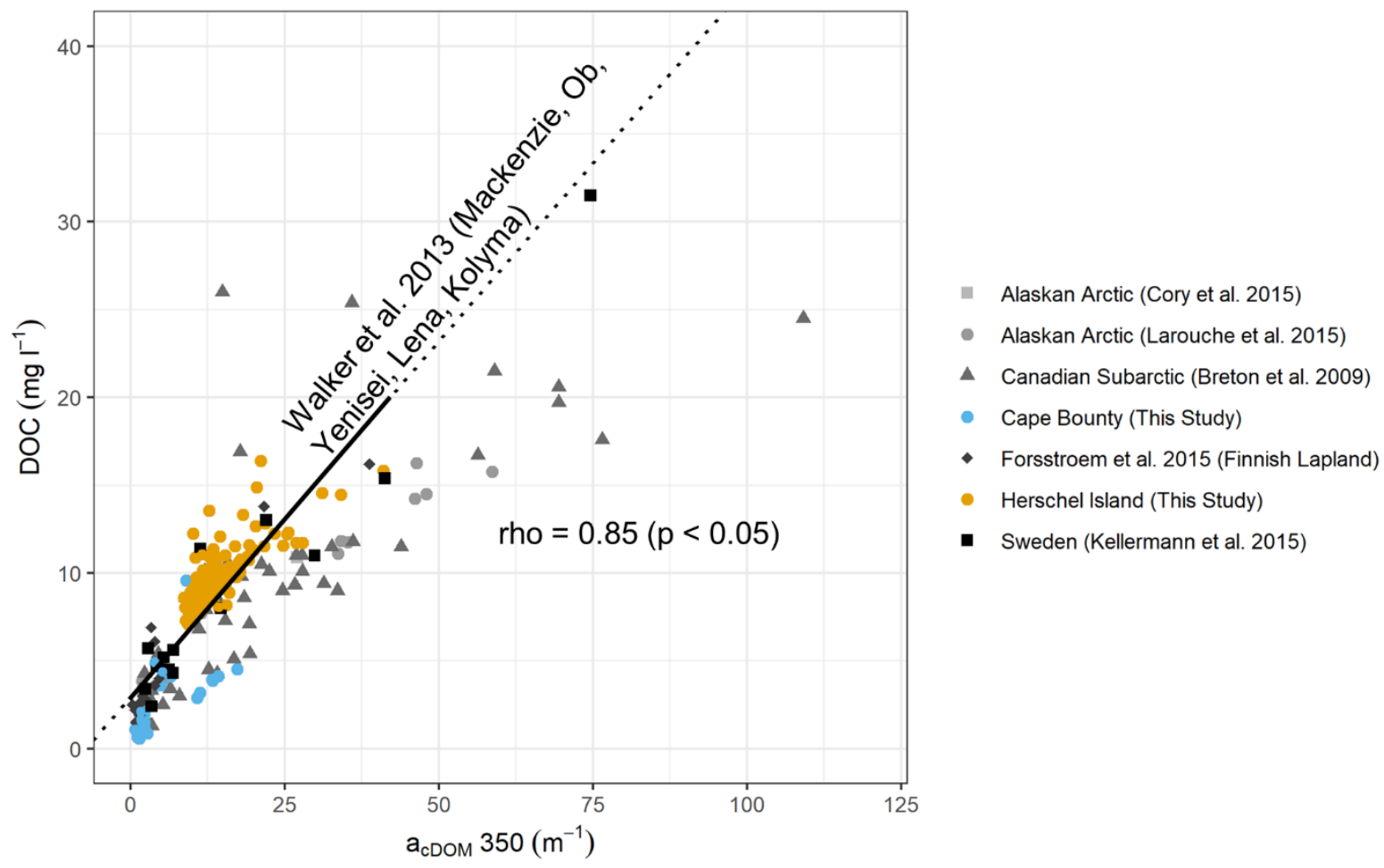

Figure S3. Relationship between acDom350 $\left(\mathrm{m}^{-1}\right)$ and DOC concentration $\left(\mathrm{mg} \mathrm{l}^{-1}\right)$ for our study sites (Herschel Island in orange and Cape Bounty in blue) and sites retrieved from the literature. The black lines represent the regression line established for the large Arctic rivers by Walker et al. (2013). The solid section marks the validity ranges for the relationship established, whereas the dotted 5 line is the linear continuation. 


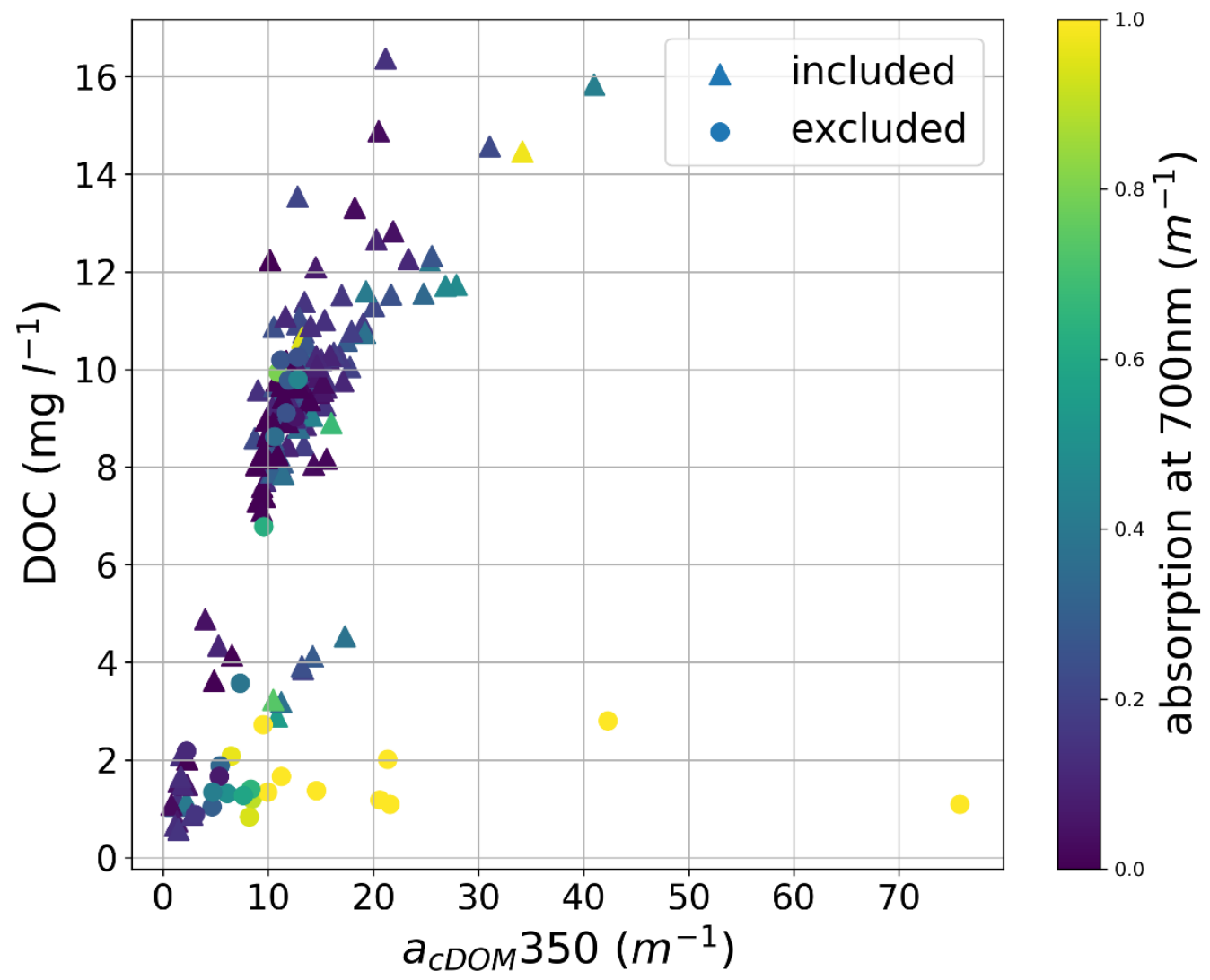

Figure S4: cDOM350 vs. DOC, colors indicate the absorption values at $700 \mathrm{~nm}$. 
Table S1. Number of samples available for different parameters (acDoм350, DOC, SUVA, S275-295, SR) retrieved from the literature.

\begin{tabular}{|l|l|l|l|l|l|l|}
\hline Study & Location & acDOM350 & DOC & SUVA & S275-295 & SR \\
\hline Breton et al. (2009) & Boniface River, forest tundra & 6 & 6 & n.a. & n.a. \\
\cline { 2 - 6 } & Bylot Island, Arctic tundra & 15 & 15 & n.a. & n.a. \\
\cline { 2 - 6 } & $\begin{array}{l}\text { Umiujaq, Sheldrake River, shrub } \\
\text { tundra }\end{array}$ & 16 & 16 & n.a. & n.a. \\
\cline { 2 - 6 } & $\begin{array}{l}\text { Whapmagoostui-Kuujuarapik, } \\
\text { forest tundra }\end{array}$ & 11 & 11 & n.a. & n.a. \\
\hline Cory et al. (2015) & Imnavait Creek & & & n.a. \\
\hline Dvornikov et al. (2018) & Yamal Peninsula & 1 & 1 & 1 & n.a. \\
\hline Forsström et al. (2015) & Finnish Lapland & 75 & n.a. & n.a. & 74 \\
\hline Kellerman et al. (2015) & Sweden & 113 & 113 & 113 & n.a. \\
\hline Skorospekhova et al. (2016) & Yamal Peninsula & 85 & n.a. & n.a. & 85 \\
\hline Skorospekhova et al. (2017) & Lena River Delta & 104 & n.a. & n.a. & 104 & n.a. \\
\hline This Study & Cape Bounty & 29 & 51 & 28 & 29 & 85 \\
\cline { 2 - 6 } & Herschel Island & $\mathbf{6 0 2}$ & $\mathbf{3 6 8}$ & $\mathbf{2 8 9}$ & $\mathbf{4 3 6}$ \\
\hline Total & & 134 & 126 & 126 \\
\hline
\end{tabular}


Table S2. Correlation matrix using the Spearman's rho correlation coefficient between DOC, optical parameters and latitude, soil organic carbon content in $30 \mathrm{~cm}$ depth and $100 \mathrm{~cm}$ depth (Hugelius et al., 2013).

\begin{tabular}{|c|c|c|c|c|c|c|c|c|}
\hline & Latitude & $\mathrm{a}_{\mathrm{cDOM}} 350$ & DOC & S275_295 & SR & SUVA & $\begin{array}{l}\text { SOCC } \\
30 \mathrm{~cm}\end{array}$ & $\begin{array}{l}\text { SOCC } \\
100 \mathrm{~cm}\end{array}$ \\
\hline Latitude & 1.00 & $\underline{-0.22}$ & -0.13 & $\underline{0.32}$ & $\underline{0.33}$ & $\underline{0.23}$ & $\underline{-0.19}$ & $\underline{-0.26}$ \\
\hline $\mathrm{a}_{\mathrm{cDOM}} 350$ & & 1.00 & $\underline{0.85}$ & $\underline{-0.63}$ & $\underline{-0.71}$ & $\underline{0.70}$ & $\underline{0.26}$ & $\underline{0.34}$ \\
\hline DOC & & & 1.00 & $\underline{-0.22}$ & $\underline{-0.52}$ & $\underline{0.50}$ & $\underline{0.53}$ & $\underline{0.51}$ \\
\hline S275_295 & & & & 1.00 & $\underline{0.70}$ & $\underline{-0.45}$ & $\underline{-0.23}$ & $\underline{-0.25}$ \\
\hline SR & & & & & 1.00 & $\underline{-0.58}$ & $\underline{-0.23}$ & $\underline{-0.57}$ \\
\hline SUVA & & & & & & 1.00 & $\underline{0.52}$ & $\underline{0.45}$ \\
\hline SOCC $30 \mathrm{~cm}$ & & & & & & & 1.00 & 0.71 \\
\hline $\begin{array}{l}\text { SOCC } \\
100 \mathrm{~cm}\end{array}$ & & & & & & & & 1.00 \\
\hline
\end{tabular}




\section{References}

Breton, J., Prairie, Y., Vallières, C., and Laurion, I.: Limnological properties of permafrost thaw ponds in northeastern Canada, Can. J. Fish. Aquat. Sci., 66, 1635-1648, 10.1139/f09-108, 2009.

Coch, C., Lamoureux, S. F., Knoblauch, C., Eischeid, I., Fritz, M., Obu, J., and Lantuit, H.: Summer rainfall DOC, solute and sediment fluxes in a small Arctic coastal catchment on Herschel Island (Yukon Territory, Canada), Arctic Science, 10.1139/as-2018-0010, 2018.

Cory, R. M., Harrold, K. H., Neilson, B. T., and Kling, G. W.: Controls on dissolved organic matter (DOM) degradation in a headwater stream: the influence of photochemical and hydrological conditions in determining light-limitation or substrate-limitation of photodegradation, Biogeosciences, 12, 6669-6685, 10.5194/bg-12-6669-2015, 2015.

10 Dvornikov, Y., Leibman, M., Heim, B., Bartsch, A., Herzschuh, U., Skorospekhova, T., Fedorova, I., Khomutov, A., Widhalm, B., Gubarkov, A., and Rößler, S.: Terrestrial CDOM in Lakes of Yamal Peninsula: Connection to Lake and Lake Catchment Properties, Remote Sensing, 10, 10.3390/rs10020167, 2018.

Forsström, L., Rautio, M., Cusson, M., Sorvari, S., Albert, R.-L., Kumagai, M., and Korhola, A.: Dissolved organic matter concentration, optical parameters and attenuation of solar radiation in high-latitude lakes across three vegetation zones, Ecoscience, 22, 17-31, $10.1080 / 11956860.2015 .1047137,2015$.

Hugelius, G., Tarnocai, C., Broll, G., Canadell, J. G., Kuhry, P., and Swanson, D. K.: The Northern Circumpolar Soil Carbon Database: spatially distributed datasets of soil coverage and soil carbon storage in the northern permafrost regions, Earth System Science Data, 5, 3-13, 10.5194/essd-5-3-2013, 2013.

Kellerman, A. M., Kothawala, D. N., Dittmar, T., and Tranvik, L. J.: Persistence of dissolved organic matter in lakes related to its molecular characteristics, Nat. Geosci., 8, 454-457, 10.1038/ngeo2440, 2015. 